\title{
论文
}

\section{表面催化特性对火星进入气固耦合热效应的 影响研究}

\author{
杨肖峰 ${ }^{1}$, 桂业伟 ${ }^{1,2^{*}}$, 刘䂞 ${ }^{2}$, 唐伟 ${ }^{2}$, 杜雁霞 ${ }^{1,2}$ \\ 1. 中国空气动力研究与发展中心, 空气动力学国家重点实验室, 绵阳 621000 ; \\ 2. 中国空气动力研究与发展中心, 计算空气动力研究所, 绵阳 621000 \\ * E-mail: guiyewei@cardc.cn \\ 收稿日期: 2017-07-13; 接受日期: 2017-11-24; 网络版发表日期: 2018-02-09 \\ 国家自然科学基金(批准号: 11702311, 11472295)资助项目
}

\begin{abstract}
摘要 高超声速进入火星大气层过程特殊且复杂的高温真实气体效应及气固界面热化学作用给火星进入器耦合 气动热环境的精准预测带来巨大挑战. 针对火星进入气固耦合问题, 建立非平衡气动热环境和结构热响应的耦合 计算方法, 开展进入器防热大底高超声速非平衡气动加热和结构传热的耦合计算分析, 获得了典型弹道点上气固 界面非平衡热化学作用对耦合加热的影响规律. 耦合分析表明, 壁温梯度所致的对流热流和组分扩散热流均对高 超声速非平衡气动加热有较大影响; 当前耦合计算状态下组分扩散热流占总热流的主要部分, 尤其 $\mathrm{CO}_{2}$ 部分影响 最大; 所计算的热防护系统能够有效阻止气动热向舱内传递，防隔热性能良好，表面热流与辐射散热可快速趋于 局部热辐射平衡, 可采用辐射平衡壁面条件解耦模拟气动热环境; 完全非催化/完全催化壁面热流随耦合时间逐 渐降低，但有限速率催化热流因界面非平衡热化学效应而先增后降; 壁面热流与壁温的线性度因界面非平衡热 化学所致扩散热流的引入而减弱.
\end{abstract}

关键词 气动加热, 结构传热, 耦合计算, 火星进入器, 表面热化学, 催化加热

\section{1 引言}

随着中国火星探测任务的不断推进 ${ }^{[1,2]}$, 进入火星 大气飞行器热防护系统的结构轻量化和安全可靠性设 计亟需准确可靠的气动热环境预测数据, 以建立可抵 抗强烈气动热冲击的热防护系统 ${ }^{[3]}$. 纵观美国历次成 功的火星进入任务 ${ }^{[4]}$, 首次着陆任务海盗号环绕火星 数圈后以较低速度进入大气层; 探路者号直接以较高
速度进入，带来更为严峻的高温真实气体效应主导的 气动加热问题; 而最新着陆的火星科学实验室尺寸更 大, 产生包括湍流在内的更复杂的非平衡表面热化学 和气固界面耦合加热效应. 进入器所采用的SLA-561V 等新型防热材料暴露于稀薄和非平衡效应主导的高焓 离解环境中, 在飞行器表面呈现由高焓离解火星气体 主导的化学非平衡、碳氧复合催化及其反应/扩散、 气/表/固相互作用等物理化学效应. 上述现象及其耦合

引用格式: 杨肖峰, 桂业伟, 刘否, 等. 表面催化特性对火星进入气固耦合热效应的影响研究. 中国科学: 技术科学, 2018, 48: 939-949 Yang X F, Gui Y W, Liu L, et al. Influence of surface catalysis on coupled aerodynamic heating for Mars entries (in Chinese). Sci Sin Tech, 2018, 48: 939-949, doi: 10.1360/N092017-00228 
热效应对高超声速气动热环境的精确预测有重要影 响, 基于单独的流场分析难以表征, 而需要通过火星进 入气动热环境和结构热响应的耦合研究予以解决.

由于火星进入问题非空气介质的特殊性，基于地 面或飞行试验的耦合热效应研究技术难度大且试验成 本高, 所以数值模拟是解决上述问题的主要研究手段. 针对地球再入环境, 已有许多学者开展高超声速气动 热和结构传热相互作用的耦合计算研究 ${ }^{[5 \sim 7]}$. 尽管可借 鉴地球环境的相关研究方法, 但探测器身处火星大气 环境的高焓非平衡碳氧环境, 而对此类环境气固界面 热化学现象和耦合作用机制的认识尚未完全清楚，亟 需针对高焓碳氧环境的气固界面热化学效应开展耦合 研究.

关于火星进入问题的耦合研究，Chen等人 ${ }^{[8]}$ 通过 复杂界面平衡关系将耦合方法运用到火星进入器气动 和防热设计中, 并通过流场求解器和烧蚀传热程序的 联合求解获得沿进入轨道的二维耦合分析结果. Milos 等人 ${ }^{[9]}$ 使用了不同的流场求解器计算探路者号进入器 高超声速流场和热防护结构热响应，获得气动加热与 防热材料的相互作用，来实现热防护系统防热特性的 更精确预测, 进而评估进入器热防护系统厚度. 在国 内，有学者将多层防热结构热传导与高超声速流场联

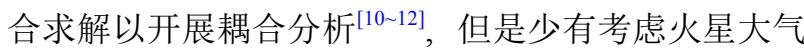
环境化学非平衡、表面催化作用等真实气体效应的耦 合热效应分析.

考虑到火星进入环境基于碳氧反应的强真实气体 效应, 本文以探路者号进入器为研究对象, 开展其高超 声速化学非平衡气动热和结构传热的耦合计算研究, 分析表面催化复合主导的扩散热流在气动热环境和结 构传热耦合过程中扮演的角色，获得多种壁面热化学 效应影响下的耦合加热机理, 为进入器热防护系统设 计提供理论依据.

\section{2 计算方法和算例验证}

\section{1 气固界面模型和耦合方法}

耦合计算涉及气固两个区域以及高超声速流场和 固体区域结构温度场等两个物理场，分别由高超声速 非平衡流动和固体结构热传导等两个计算模块来完 成，并通过气固界面上的能量平衡关系来实现各计算 模块的自封闭 ${ }^{[13]}$.
针对常规尺度的高超声速进入问题，忽略界面上 的温度跳跃和接触热阻效应，并考虑界面上热流通量 连续. 高超声速飞行器在气固界面上有多种能量分配 和传递方式 ${ }^{[14]}$ ，重点考虑流场的对流加热与组分扩散 加热、固体结构导热以及表面向空间的辐射散热, 因 而界面上的热流满足如下界面平衡方程:

$q_{\mathrm{w}}^{\text {conv }}+\sum_{s=1}^{N_{s}} q_{\mathrm{w}, s}^{\text {diff }}=q_{\mathrm{w}}^{\text {cond }}+q_{\mathrm{w}}^{\text {rad }}$,

式中, 组分 $s$ 的扩散热流与辐射散热热流分别为

$q_{\mathrm{w}, s}^{\mathrm{diff}}=\rho D_{s} h_{s} \frac{\partial c_{s}}{\partial n}$,

$q_{\mathrm{w}}^{\mathrm{rad}}=\varepsilon \sigma\left(T_{\mathrm{w}}^{4}-T_{\infty}^{4}\right)$,

其中, $\rho$ 为气体混合物总密度, $D$ 为等效扩散系数, $h$ 为 绝对焓即显焓和化学生成焓之和, $\varepsilon$ 为表面辐射发射 率, $\sigma$ 为玻尔兹曼常数.

由于流场的时间尺度远小于温度场的时间尺度， 所以在耦合计算过程中流场采用定常计算, 而结构温度 场采用非定常计算 ${ }^{[7,10]}$. 耦合迭代计算流程见图1. 流场 和结构场网格在气固界面上存在空间不一致性, 需要鲁 棒的界面插值程序来实现气固界面上高效精确的数据 传递. 气固界面上未知的温度和热流数据通过基于归 一化改进径向基函数法的空间插值方法来获取 ${ }^{[15]}$.

考虑到初始时刻温升较快，为确保在每一个时间 间隔里结构场温升均匀, 采用可变时间步长进行耦合 迭代, 耦合时间步长与驻点热流相关.

$\Delta t_{i}=\Delta t_{0}+k_{t} \frac{q_{\mathrm{w}, 0}-q_{\mathrm{w}, i}}{q_{\mathrm{w}, 0}-q_{\mathrm{w}, \mathrm{re}}}$,

式中, $q_{\mathrm{w}, \mathrm{re}}$ 为驻点辐射平衡热流, $q_{\mathrm{w}, 0}$ 为初始时刻驻点热 流, 取耦合初始时间步长 $\Delta t_{0}=1 \times 10^{-5} \mathrm{~s}$, 时间步长斜率 $k_{t}=0.02 \mathrm{~s}$.

\section{2 气动加热计算方法}

高超声速流场计算模块以三维可压缩化学非平衡 Navier-Stokes方程组为控制方程

$\frac{\partial \mathbf{Q}}{\partial t}+\frac{\partial \mathbf{F}}{\partial x}+\frac{\partial \mathbf{G}}{\partial y}+\frac{\partial \mathbf{H}}{\partial z}=\frac{\partial \mathbf{F}_{\mathbf{v}}}{\partial x}+\frac{\partial \mathbf{G}_{\mathbf{v}}}{\partial y}+\frac{\partial \mathbf{H}_{\mathbf{v}}}{\partial z}+\mathbf{S}$,

其中, $\mathbf{Q}$ 为守恒型状态向量, $\mathbf{F}, \mathbf{G}$ 和 $\mathbf{H}$ 为三方向的无黏 通量向量, $F_{v}, G_{v}$ 和 $H_{v}$ 为黏性通量向量, $S$ 为化学反应 源项. 


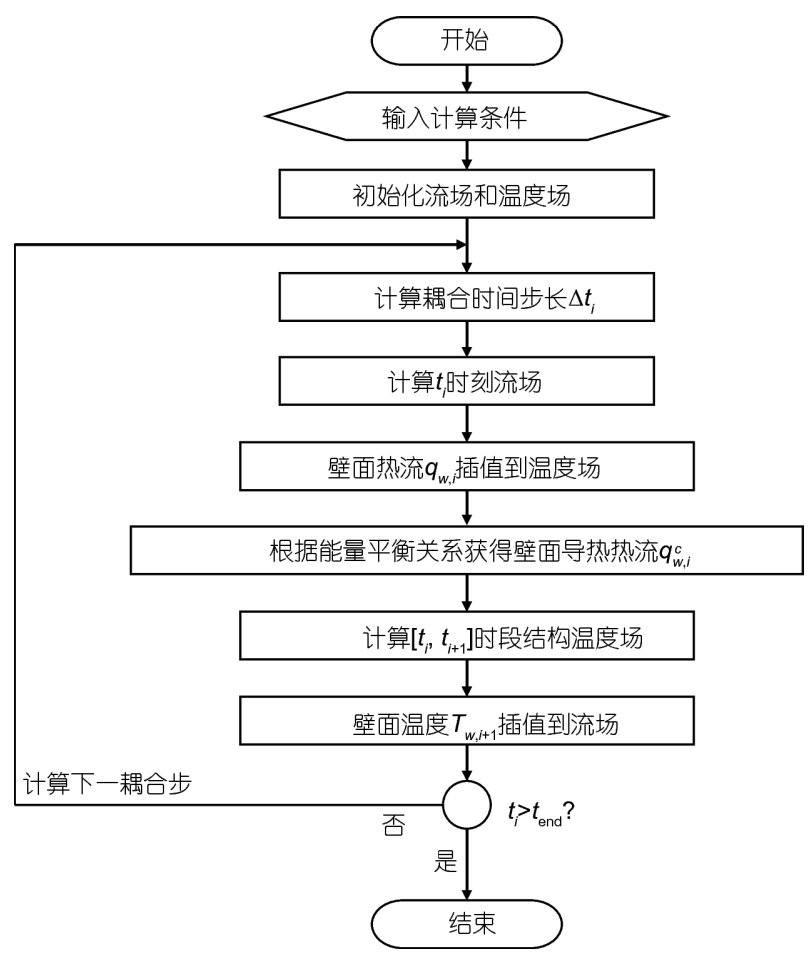

图 1 耦合计算流程

控制方程使用有限体积法进行离散. 空间对流通 量采用二阶van-Leer分裂的总变差减小(total variation diminishing, TVD)方法作矢通量分裂, 扩散通量采用 二阶中心格式, 面心的通量采用MUSCL方法(带有van Albada限制器)插值求得. 时间方向采用LU-SGS的隐 式离散方法 ${ }^{[16]}$.

由于火星大气稀薄, 进入器进入过程雷诺数较低 $\left(10^{5}\right.$ 量级), 计算只考虑层流流动 ${ }^{[17]}$. 因火星进入过程 基本处于热力学平衡和化学非平衡状态 ${ }^{[18]}$, 求解器采 用描述化学非平衡的一温度模型.

考虑火星大气的主要成分 $\mathrm{CO}_{2}$, 化学反应机制采 用基于Park化学动力学的 5 组分 6 化学反应模型, 如表 1 所示, 反应速率系数见文献[19]. 高焓离解气体各组分 热力学特性考虑分子平动能、转动能、振动能和束缚 电子激发能 ${ }^{[20]}$, 黏性系数由Blottner拟合关系式获得 ${ }^{[21]}$. 混合气体的热力学和输运系数由Wilke公式计算 ${ }^{[22]}$. 气 体组分的等效扩散系数由Schmidt数 $(S c=0.5)$ 获得.

\section{3 结构传热计算方法}

结构温度场计算模块以非定常的热传导方程为控
表 1 火星气体化学反应机制 ${ }^{[19]}$

\begin{tabular}{ccc}
\hline 编号 & 化学反应方程 & 第三体反应物 \\
\hline $\mathrm{R} 1$ & $\mathrm{CO}_{2}+\mathrm{M}_{1}<=>\mathrm{CO}+\mathrm{O}+\mathrm{M}_{1}$ & $\mathrm{C} / \mathrm{O} / \mathrm{O}_{2} / \mathrm{CO} / \mathrm{CO}_{2}$ \\
$\mathrm{R} 2$ & $\mathrm{CO}+\mathrm{M}_{2}<=>\mathrm{C}+\mathrm{O}+\mathrm{M}_{2}$ & $\mathrm{C} / \mathrm{O} / \mathrm{O}_{2} / \mathrm{CO} / \mathrm{CO}_{2}$ \\
$\mathrm{R} 3$ & $\mathrm{O}_{2}+\mathrm{M}_{3}<=>\mathrm{O}+\mathrm{O}+\mathrm{M}_{3}$ & $\mathrm{C} / \mathrm{O} / \mathrm{O}_{2} / \mathrm{CO} / \mathrm{CO}_{2}$ \\
$\mathrm{R} 4$ & $\mathrm{CO}_{2}+\mathrm{O}<=>\mathrm{O}_{2}+\mathrm{CO}$ & - \\
R5 & $\mathrm{CO}+\mathrm{O}<=>\mathrm{O}_{2}+\mathrm{C}$ & - \\
R6 & $\mathrm{CO}+\mathrm{CO}<=>\mathrm{CO}_{2}+\mathrm{C}$ & - \\
\hline
\end{tabular}

制方程

$\rho C_{\mathrm{p}} \frac{\partial T}{\partial t}=\frac{\partial}{\partial x}\left(\kappa \frac{\partial T}{\partial x}\right)+\frac{\partial}{\partial y}\left(\kappa \frac{\partial T}{\partial y}\right)+\frac{\partial}{\partial z}\left(\kappa \frac{\partial T}{\partial z}\right)$,

其中, $T$ 为固体结构场温度变量, $\rho$ 为结构密度, $C_{\mathrm{p}}$ 为结 构比热, $\kappa$ 为结构导热系数. 热物性参数 $\rho, C_{\mathrm{p}}, \kappa$ 均为 $T$ 的 函数.

使用有限体积法对扩散型热传导方程进行离散, 其中空间方向使用二阶中心格式离散，时间推进使用 二阶TVD-Runge-Kutta方法离散 ${ }^{[23]}$. 因计算状态烧蚀 量较小, 故暂不考虑气动加热带来的结构内部烧蚀和 热解作用, 仅分析表面热化学效应和结构内部热传导 作用.

\section{4 非平衡气动加热和耦合方法的验证}

采用两种风洞测试条件下的LENS-I反射激波风 洞高超声速球头绕流实验和兰利高温风洞高超声速圆 管绕流实验，分步验证本文程序在非平衡火星大气气 动加热和耦合计算方法方面的有效性.

LENS-I反射激波风洞高超声速球头绕流实验模 型为76.2 $\mathrm{mm}$ 的球头并以 $\mathrm{CO}_{2}$ 为测试工质 ${ }^{[24]}$. 计算所得 的高超声速流动符合预期, 流场结构正确可信. 图2给 出完全催化壁条件下冷壁 $(300 \mathrm{~K})$ 热流分布 (calc fcw) 及与实验值 $(\exp ) 、 \mathrm{NASA}$ 高温化学反应流动求解器 DPLR计算结果 (ref fcw)的对比, 本程序计算值和 DPLR值吻合良好, 驻点区域计算结果略低于实验值, 远离驻点区域二者吻合较好. 表明使用该方法可获得 可信的火星环境高超声速气动加热值.

根据NASA兰利研究中心高温风洞的不锈钢圆管 (内/外径 $50.8 / 76.2 \mathrm{~mm}$ ) 实验数据及Dechaumphai耦合 计算结果开展本文耦合计算方法的有效性验证 ${ }^{[7]}$, 其 中来流马赫数 6.47 , 测试工质为空气. 在常温 $294.4 \mathrm{~K}$ 


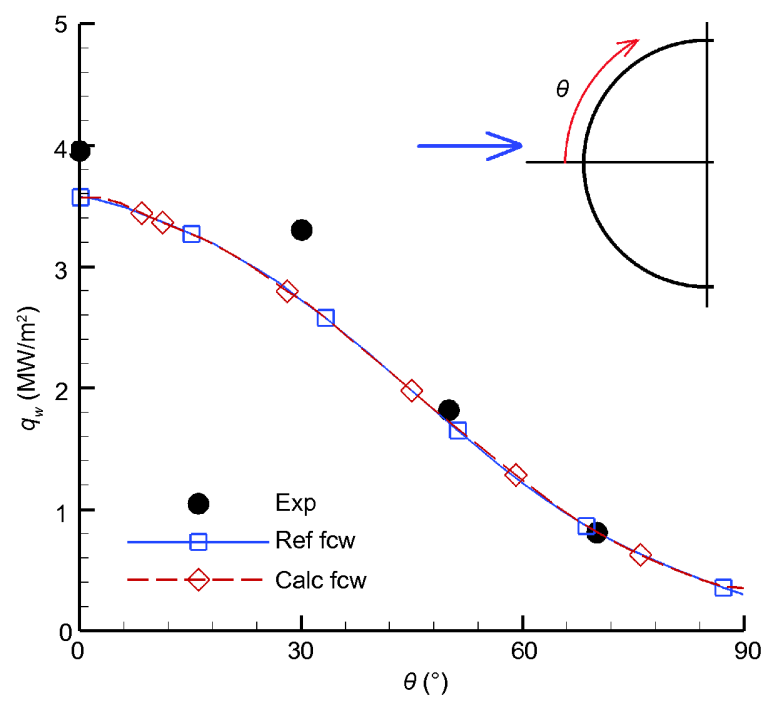

图 2 (网络版彩图) $\mathrm{CO}_{2}$ 气体非平衡气动加热验证算例

的初始条件下, 高超声速流动产生 $491 \mathrm{~kW} / \mathrm{m}^{2}$ 的冷壁驻 点热流，比文献热流值 $484 \mathrm{~kW} / \mathrm{m}^{2}$ 略高. 在加热 $2 \mathrm{~s}$ 后, 壁温逐渐升高至 $391 \mathrm{~K}$ ，略高于文献值 389 K，同时壁 面热流也随之降低. 图3为计算所得的归一化壁面热 流分布(calc)及与文献值(ref)和实验值(exp)的对比，两 个时刻 $(t=0,2 \mathrm{~s})$ 的热流分布与文献值吻合度较好, 进 而验证了本耦合计算方法的有效性.

\section{3 进入器模型与网格}

\section{1 进入器布局和热防护结构}

美国历次成功着陆火星表面的进入器气动布局均 由球雉型防热大底和倒雉型后体组成, 防热大底头部 半径为参考长度 (最大截面直径)的 $1 / 4$, 半雉角 $70^{\circ[25]}$. 以进入热环境较为严重的探路者号进入器的防热大底 为研究对象, 因外形尺寸较小, 进入雷诺数较低, 计算 忽略湍流因素. 进入器按弹道式(零攻角)进入火星大 气层, 并针对火星表面高度 $28.5 \mathrm{~km}$ 、进入速度 $4862 \mathrm{~m} / \mathrm{s}$ 的弹道点开展耦合计算研究 ${ }^{[26]}$, 其进入攻角 和侧滑角均为零. 此次进入任务的防热大底结构是由 SLA-561V轻质微烧蚀材料、铝蜂窝及夹层结构等组成 的多层结构, 各层厚度分别为 $19.95,31.75$ 和 $0.38 \mathrm{~mm}^{[0]}$. 多层防热材料的热物性参数见文献[27]. 图4给出了进 入器防热大底热防护结构组成示意图.

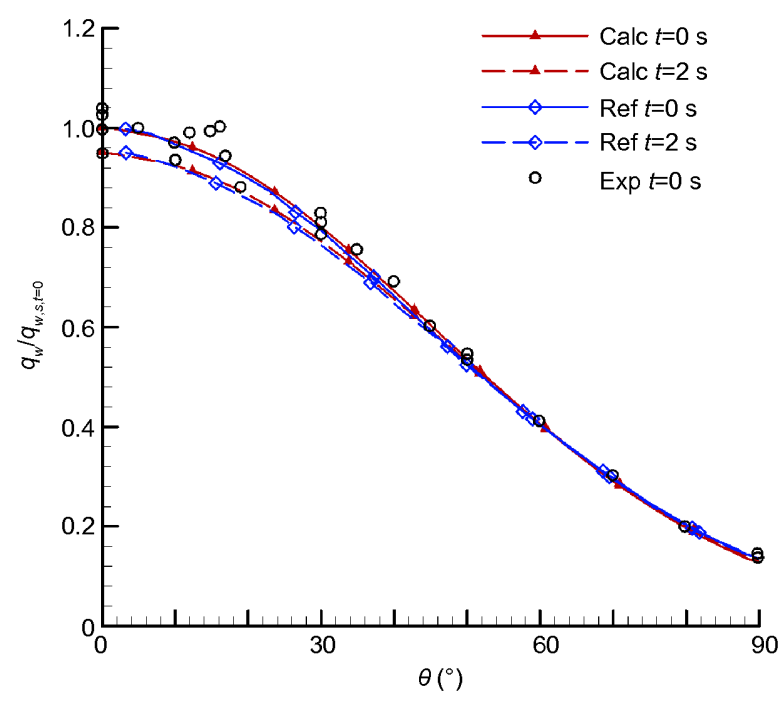

图 3 (网络版彩图)耦合气动加热验证算例

\section{2 计算网格和边界/界面条件}

流体区域计算网格为三维多块六面体结构网格, 壁面法向足够正交且适当加密, 保证首层网格单元雷 诺数 $R e_{\mathrm{c}}=\mathrm{O}(1)$, 以确保模拟出复杂的热边界层流动并 获得具有较高网格无关性的精确热流值. 固体区域为 三维非结构化的六面体网格, 靠近气固界面处适当加 密, 以保证较大温度梯度区域的预测精度.

流场区域远场采用自由流条件, 对称面采用对称 条件, 流场出流处采用超声速外插条件; 气固界面考 虑完全非催化(ncw)、完全催化(fcw)和有限速率催化 (FR)壁面条件并采用上述耦合计算方法实现各模块自 封闭; 固体区域其他各面采用绝热条件. 有限速率催化 壁采用如表2所示的表面吸附/Eley-Rideal催化反应机 制 $^{[28]}$. 第 $r$ 个吸附/催化反应速率为

$k_{r}=\frac{\bar{v}_{r}}{4 \Phi_{r}} \gamma_{r} \exp \left(-\frac{E_{r}}{R_{0} T}\right)$,

其中, $\Phi_{r}$ 为吸附位或吸附组分浓度, $\gamma_{r}$ 为黏附系数(吸 附)或催化效率(Eley-Rideal), $E_{r}$ 为吸附或催化活化能, $R_{0}$ 为通用气体常数, 而粒子运动平均热速度为

$\bar{v}=\sqrt{\frac{8 R_{0} T_{\mathrm{w}}}{\pi M}}$,

其中, $M$ 为相关分子或原子的相对分子量. 本文计算假 设吸附位充足, 黏附系数或催化效率为 $\gamma_{r}=1$, 吸附或 催化活化能为 $E_{r} / R_{0}=2800 \mathrm{~K}$. 

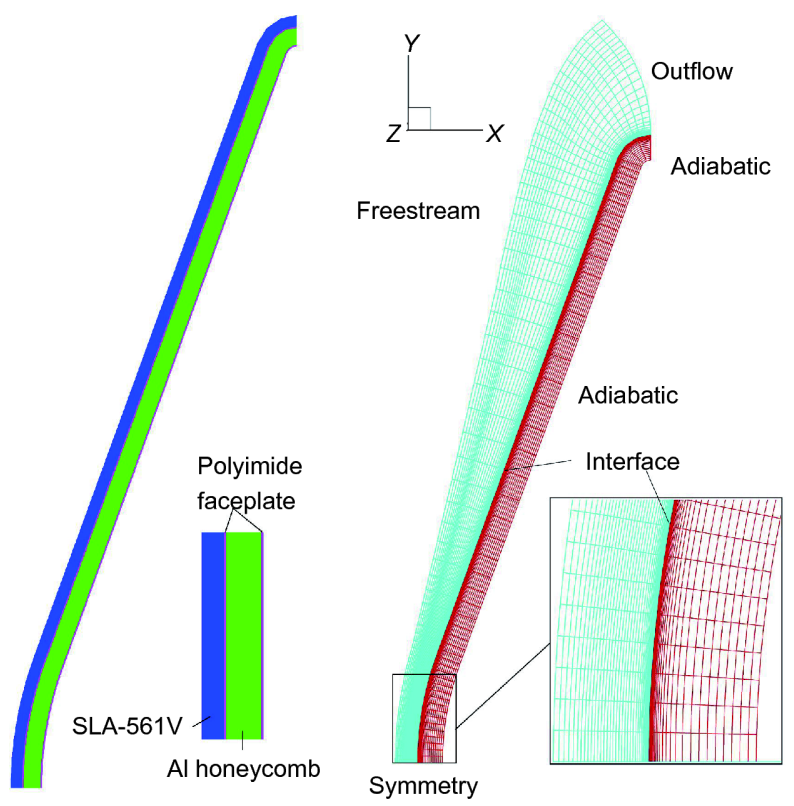

图 4 (网络版彩图)热防护结构、计算网格和边界条件

表 2 表面吸附/催化反应机制[27]

\begin{tabular}{ccc}
\hline 编号 & 吸附/催化反应方程 & 反应类型 \\
\hline $\mathrm{Ad} 1$ & $\mathrm{O}+(\mathrm{s}) \rightarrow \mathrm{O}(\mathrm{s})$ & 吸附 \\
$\mathrm{Ad} 2$ & $\mathrm{CO}+(\mathrm{s}) \rightarrow \mathrm{CO}(\mathrm{s})$ & 吸附 \\
$\mathrm{ER} 1$ & $\mathrm{O}+\mathrm{O}(\mathrm{s}) \rightarrow \mathrm{O}_{2}+(\mathrm{s})$ & Eley-Rideal \\
$\mathrm{ER} 2$ & $\mathrm{CO}+\mathrm{O}(\mathrm{s}) \rightarrow \mathrm{CO}_{2}+(\mathrm{s})$ & Eley-Rideal \\
$\mathrm{ER} 3$ & $\mathrm{O}+\mathrm{CO}(\mathrm{s}) \rightarrow \mathrm{CO}_{2}+(\mathrm{s})$ & Eley-Rideal \\
\hline
\end{tabular}

图4还给出了进入器防热大底流动区域和热防护 结构的计算网格和边界/界面条件. 另外采用等壁温/辐 射平衡及完全非/完全催化壁面条件研究壁面条件对 进入器非平衡流场及气动加热的影响规律.

\section{4 壁面条件对进入器非平衡流场及气动加 热的影响}

暂不考虑耦合热效应，开展固定壁面条件下的高 超声速非平衡流动的数值模拟, 获得火星进入器高超 声速进入过程的典型流场结构，研究壁面条件对进入 器非平衡流场及气动加热的影响规律.

自由来流以极高速度撞击到防热大底表面，气体 的强压缩性导致远离防热大底的很小一段距离处形成
弓形脱体激波. 激波层内流动表现出较强的真实气体 效应, 具体表现为: $\mathrm{CO}_{2}$ 来流的大规模离解、化学反应 所致的非平衡效应及非平衡离解组分壁面复合产生的 催化加热作用.

基于多种壁面组分和热边界条件的数值计算表 明，壁面条件对火星进入器高超声速流场结构的影响 较弱，但近壁面的温度和组分分布规律因壁面条件不 同而迥异，因而产生相差甚大的壁面热流. 图5给出了 考虑完全非催化/完全催化壁条件以及冷壁温 $(300 \mathrm{~K})$ /辐射平衡(rad-eq, $\varepsilon=0.78$ )条件防热大底壁面热流分 布曲线. 壁面温度条件方面, 冷壁温条件造成较大的壁 面法向温度梯度, 故壁面热流较高; 而辐射平衡条件壁 温较高, 壁面热流相对较低. 壁面组分条件方面, 完全 非催化壁条件下, 壁面无复合防热作用, 热流全部来源 于壁面法向温度梯度, 气动加热量较低; 而完全催化壁 面热流除了壁面法向温度梯度所致的对流加热外，还 包含大量的复合催化加热. 因完全催化条件强制气体 混合物化学焓在壁面降至最低，促使能量全部以化学 生成焓的形式在壁面释放，催化复合作用带来的组分 扩散热流成为非平衡气动加热的重要组成部分.

火星进入过程较强的真实气体效应造成扩散热流 不可忽视, 甚至对气动热环境有主导性作用. 另外, 气 固界面非平衡化学热对处理变壁温对流加热的热壁修 正方法的适用性有待研究. 流动的非平衡性和表面热化 学效应以及壁面温度和组分提法的不确定性促使我们

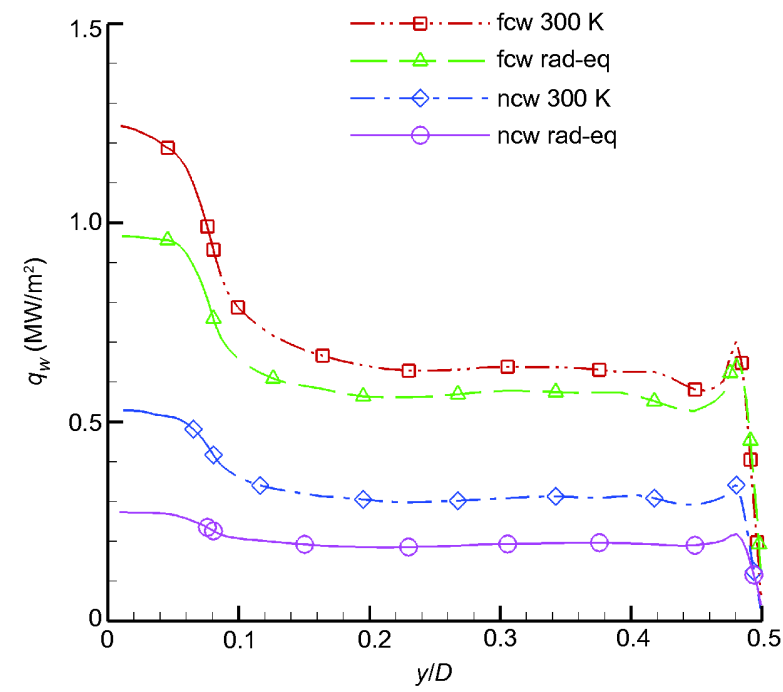

图 5 (网络版彩图)不同物面条件下防热大底物面热流分布 曲线 
开展考虑火星大气非平衡气动加热的耦合计算研究.

\section{5 非平衡气动热和结构传热的耦合分析}

\section{1 耦合作用下的驻点热流和温度}

考虑壁面完全催化作用，开展高超声速流场和结 构温度场的耦合计算研究. 考虑到初始时刻温升较快, 采用可变时间步长按照给定的耦合计算流程开始耦合 计算.

防热大底结构全场给定初始温度 $300 \mathrm{~K}$, 在该壁温 条件下计算进入器初始的高超声速流场, 获得 $1.27 \mathrm{MW} / \mathrm{m}^{2}$ 的初始驻点热流. 图6给出了耦合计算条 件下驻点热流和温度随耦合时间的变化趋势. 随着耦 合过程的推进，热防护系统表面被加热，驻点热流逐 渐降低, 而驻点温度逐渐升高. 防热结构充分加热后, 壁面热流和温度逐渐趋于辐射平衡热流和温度.

在初始 $300 \mathrm{~K}$ 冷壁温的条件下, 壁面热流最大, 随 后防热结构被迅速加热, 热流迅速降低. 图7给出了气 固区域对称轴线上的温度剖面. 由于存在较高的冷壁 热流，防热结构外表面温度迅速升高，并在气固界面 附近固体区域侧形成较高的温度梯度，且随时间推进 而逐渐向结构深度方向扩散，其扩散速率与防热材料 的热物性相关.

在流体和固体区域内存在激波间断、波后非平衡 温度梯度、近壁面热边界层、结构区域靠近界面处的 热扩散等4个高温度梯度区域(图7). 图8进一步给出了 $2 \mathrm{~s}$ 时刻气固区域对称面上的温度分布云图，气动加热

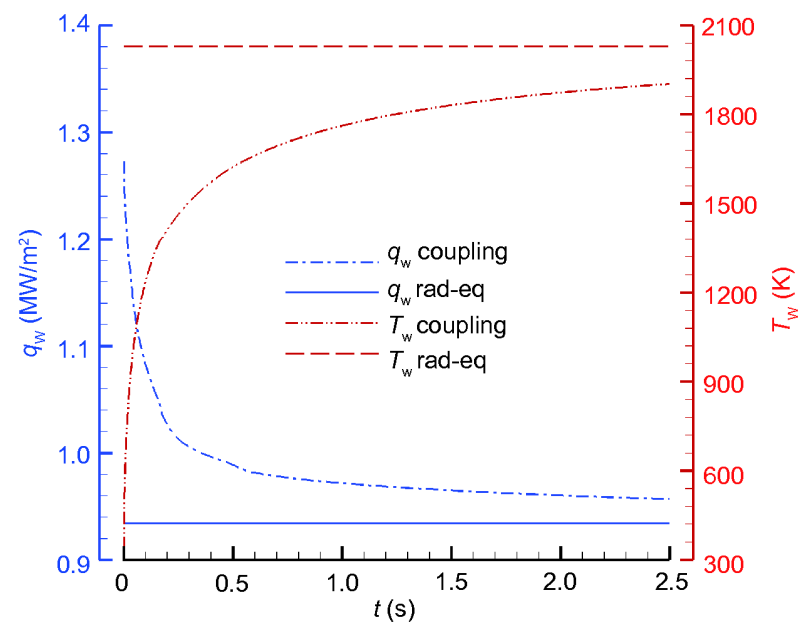

图 6 (网络版彩图)耦合过程壁面温度和热流变化趋势

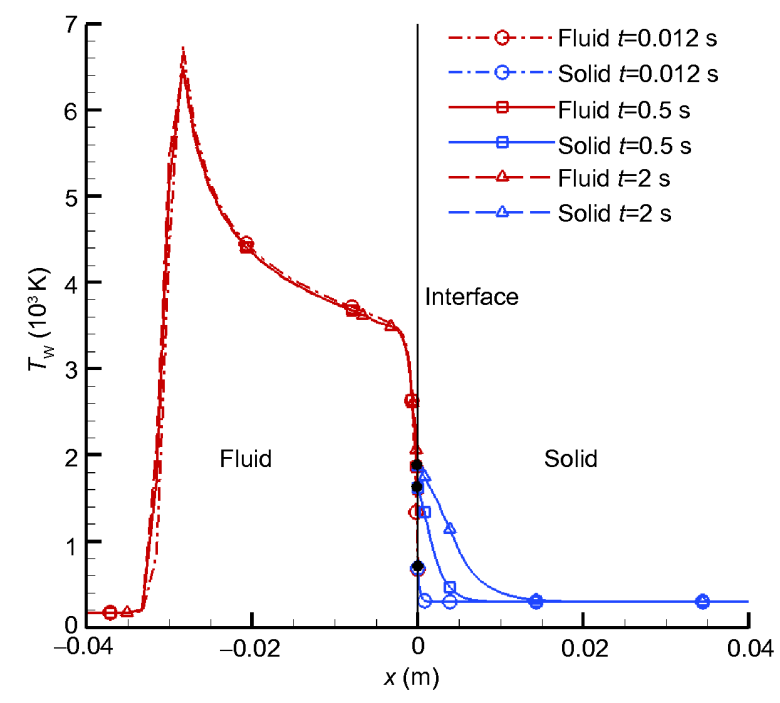

图 7 (网络版彩图)流固区域对称轴线上的温度分布

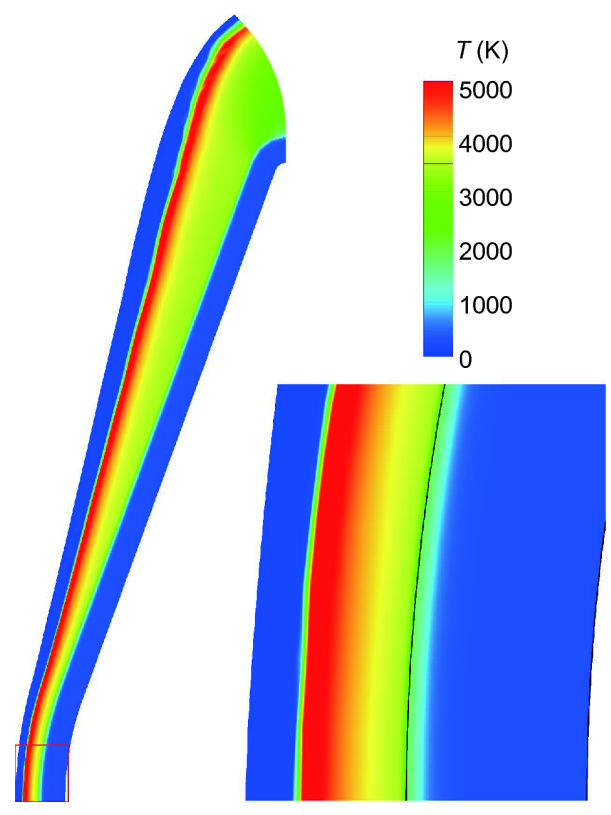

图 8 (网络版彩图)流固区域对称面上的温度分布云图(2s)

导入结构内部的热量累积量显著.

\section{2 界面热通量的分配与传递}

由于完全催化条件下气动加热包括近壁面法向温 度梯度所致的对流热流和近壁面组分扩散所致的扩散 热流等两部分热流，为此分别研究这两部分热流随耦 合时间的变化规律. 图9给出了耦合计算条件下驻点 
热流的对流和扩散分量随耦合时间的变化趋势. 可以 看出，在当前来流条件下组分扩散所致的热流占总热 流量的70\% $80 \%$, 且二者均随耦合时间逐渐降低. 在 耦合计算后期 $(0.5 \mathrm{~s}$ 以后 $)$, 扩散热流逐渐趋于恒定, 气 动热增量主要产生壁面法向温度梯度.

图10进一步给出了各个组分对耦合热效应的贡献 量. 可以看出, 随着耦合过程壁温的升高, $\mathrm{CO}_{2}$ 扩散热 流降低, 而 $\mathrm{O}_{2}$ 和 $\mathrm{CO}$ 扩散热流增高, $\mathrm{O}$ 扩散热流出现陡 然峰值后缓慢增高; 因C含量极少故其扩散热流基本 为零; 因为完全催化条件促使各离解组分全部复合为 $\mathrm{CO}_{2}$, 所以 $\mathrm{CO}_{2}$ 扩散热流增量与其他组分扩散热流增量 的符号相反且占主导地位.

耦合策略指出对流加热、各组分扩散加热、表面 辐射散热与结构内部导热在气固界面达到局部平衡. 为此图9还给出了耦合计算条件下驻点区域壁面辐射 散热热流和结构内导热热流随耦合时间的变化趋势, 界面上各物理过程对应的热流满足能量平衡关系式. 尽管总热流随耦合进程不断下降, 但壁面辐射散热热 流因壁温增高而增大, 而结构内导热热流将在壁面趋 于辐射平衡时趋于零.

耦合分析还认为, 对于探路者号火星进入器的热 防护系统，其采用低热导率、高空间辐射率的热防护 材料, 在较长时间内防热结构内壁面依然保持在较低 温度的水平, 有助于阻止气动热载荷向舱内传递且能 够有效地将气动加热量辐射到空间中; 低热导率的防 热大底结构导热作用较弱, 表面温升较快, 温度梯度 和组分扩散所引起的气动加热与表面辐射散热之间形 成局部热辐射平衡, 因此, 采用辐射平衡壁面条件可近 似获得符合真实物理的气动加热规律.

\section{6 气固界面热化学对耦合热效应的影响}

\section{1 不同界面热化学作用下的耦合分析}

火星进入器高超声速进入飞行伴随真实气体效 应, 造成气固界面热化学作用显著, 且界面热化学行为 对壁面温度较敏感, 为此研究不同的气固界面热化学 模型对进入器耦合热效应的影响规律.

分别考虑完全非催化(ncw)、完全催化(fcw)和有 限速率催化(fr2800)壁模型条件, 开展高超声速非平衡 气动加热和热响应的耦合计算研究. 图11(a)给出了 3 种壁面热化学条件下的耦合驻点热流随时间的变化曲

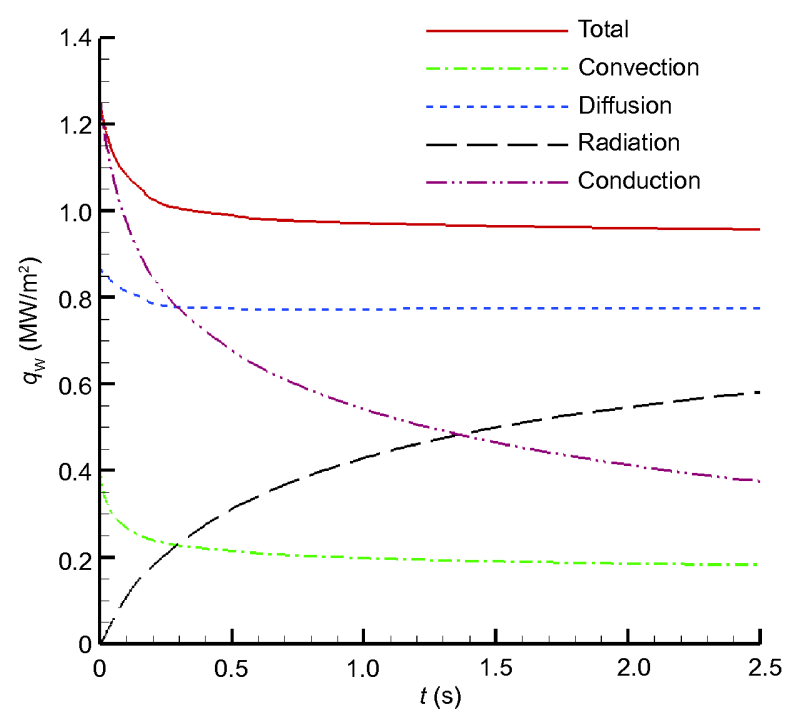

图 9 (网络版彩图)耦合过程驻点对流热流、扩散热流、结 构导热热流和辐射散热热流变化趋势

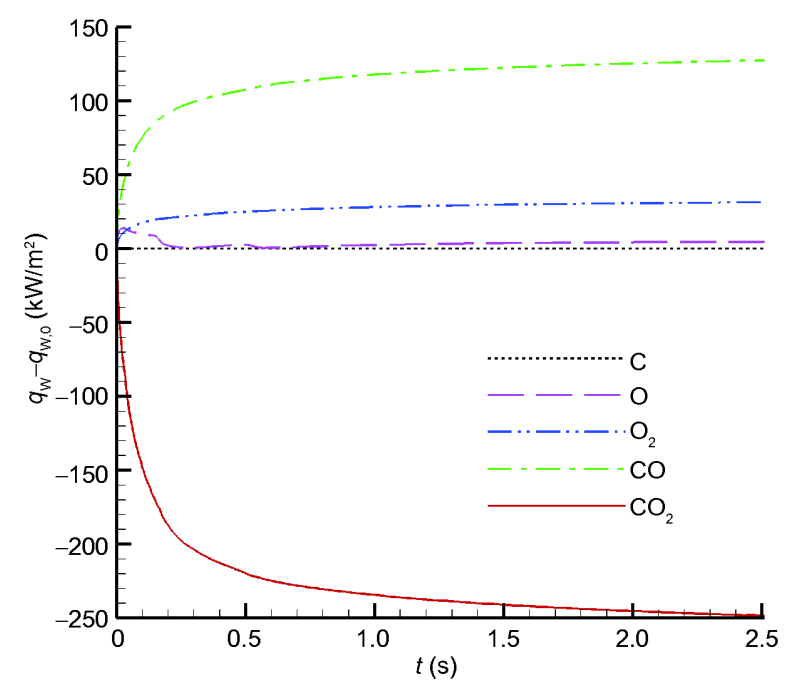

图 10 (网络版彩图)耦合过程各组分驻点扩散热流变化趋 势

线. 在初始时刻, 完全催化壁获得最高的驻点加热值, 完全非催化壁结果最低, 而催化活化能 $E_{\mathrm{r}} / R_{0}=2800 \mathrm{~K}$ 条件下的有限速率催化壁结果介于二者之间. 完全非 催化和完全催化壁面热流常规性地随耦合时间逐渐降 低, 但有限速率催化热流随耦合时间先升高后降低, 并 在当前计算条件下在 $t=0.03 \mathrm{~s}$ 时刻附近出现峰值热流. 在耦合初始时刻, 因低温条件材料的催化特性较弱, 有 限速率催化壁面热流更接近于完全非催化壁, 且随耦 合时间而逐渐升高; 在耦合计算后期, 因壁温充分升 

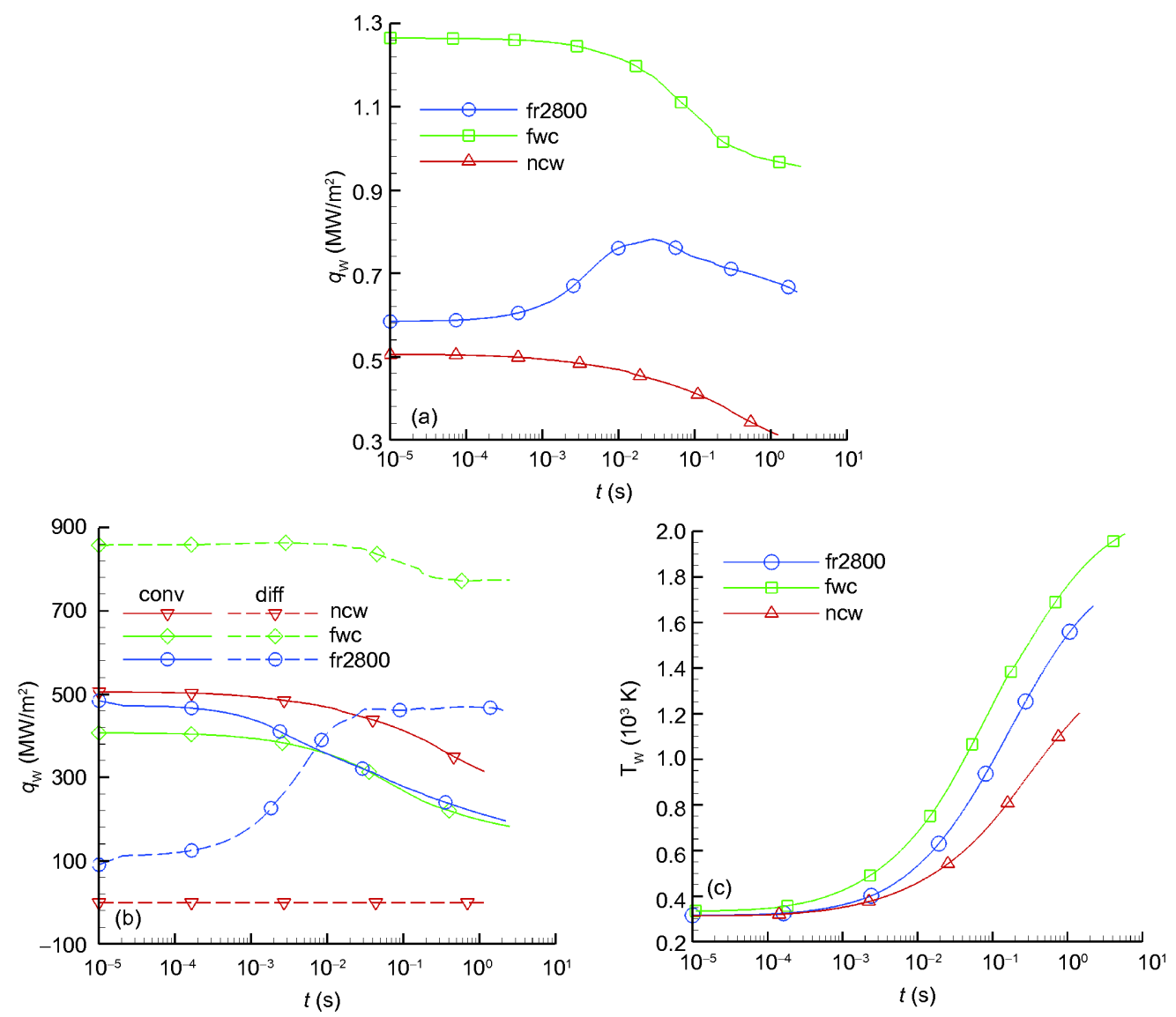

图 11 (网络版彩图)不同表面热化学条件下驻点热流分布曲线(a)、对流和扩散热流(b)和温度分布曲线(c)

高而催化放热所致扩散热流影响减弱，壁面热流随耦 合时间而逐渐降低并在耦合中间时段形成峰值热流.

为了研究气固界面热化学效应对非平衡耦合加热 的影响规律, 图11(b)给出了3种壁面热化学条件下的 驻点对流热流(conv)和扩散热流(diff)随耦合时间的变 化曲线. 可见3种催化壁条件下由温度梯度引起的对流 热流变化规律相似, 均随耦合时间而逐渐下降. 完全非 催化壁对流热流因壁面无催化放热现象而相对最高, 而完全催化条件下壁面催化放热程度最充分故壁面热 流最低. 对有限速率催化壁，在起始阶段因壁温较低, 较低温度条件下壁面催化特性较弱, 故对流热流接近 完全非催化壁结果; 相反, 在耦合后期因壁温升高, 壁 面催化特性趋于活跃, 故对流热流转而接近完全催化 壁结果. 与壁面对流热流不同，三种催化壁条件下催 化反应所致的壁面扩散热流差别甚大. 正如预期，完 全非催化壁因无壁面化学反应而扩散热流为零; 而完 全催化条件下壁面复合反应最为剧烈, 化学热释放量
最大，故扩散热流值最高；在当前条件下有限速率壁 面复合反应随壁温的升高而增强, 故由此所致的扩散 热流随之增加. 综上可知, 因表面化学反应速率的温 度依赖性较强, 故有限速率催化作用下的耦合加热规 律与完全催化或完全非催化壁结果出现明显差异.

图11(c)进一步给出了不同壁面热化学条件下的 耦合驻点温度随时间的变化曲线. 有限速率催化壁温 度介于完全非催化和完全催化壁之间, 同样因有限速 率催化作用较强的温度依赖性, 耦合初始段温度接近 于完全非催化壁, 耦合后期温度迅速爬升并远离完全 非催化壁.

\section{2 关于界面热化学作用下的壁面温度和热流的 线性度的讨论}

对完全气体气动加热问题, 工程上认为热流系数, 即斯坦顿数 $S t$ 为定值, 即相同来流条件下壁面热流与 壁面温度呈线性关系, 进而引出基于冷壁热流结果预 
测热壁气动加热量的热壁修正方法 ${ }^{[20]}$.

图12给出了3种催化壁条件下耦合计算过程驻点 热流随驻点温度的变化曲线以及其线性拟合(fit)情况. 可以看出, 随着耦合过程的发展, 完全非催化壁条件下 耦合计算过程的壁面热流随壁面温度的下降曲线有较 强的线性度(标准差 $R^{2}=0.99932$ ). 考虑壁面热化学效 应的壁面热流和温度的线性度减弱. 具体地, 完全催化 壁的热流和温度线性度降为 $R^{2}=0.98198$; 催化活化能 $E_{\mathrm{c}} / R_{0}=2800 \mathrm{~K}$ 条件下的有限速率催化壁热流和温度 线性度进一步降为 $R^{2}=0.90975$, 壁面热流和温度关系 的单调性也因壁面热化学效应而改变. 因此, 壁面非平 衡热化学效应的参与造成描述气动加热热流系数的 $S t$ 不再恒定, 处理变壁温对流加热的热壁修正方法适用 性差. 从这一点也可看出, 使用耦合计算方法对具有 复杂真实气体效应和气固界面热化学效应的火星高超 声速进入气动热环境进行数值预测是十分必要的.

\section{7 结论}

面对特殊而又复杂的火星进入器界面热化学效应 与非平衡气动加热环境, 建立高超声速非平衡气动加 热和结构传热的耦合计算方法, 并针对探路者号火星 进入器球雉型防热大底及其多层防热结构, 开展高超 声速非平衡气动加热和结构传热的耦合计算分析, 获 得火星进入器在高超声速进入过程中的典型耦合加热 规律, 主要得出以下研究结论.

(1) 壁面温度影响近物面法向梯度进而改变对流 热流, 而表面催化效应影响近物面组分扩散进而改变 扩散热流, 二者均对高超声速非平衡气动加热有较大 影响.

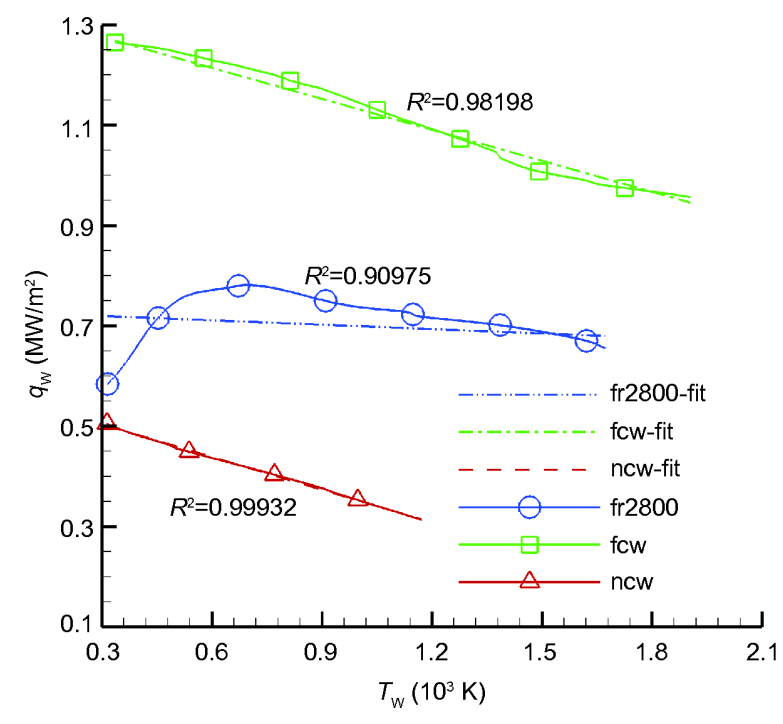

图 12 (网络版彩图)不同表面催化壁条件下驻点热流和温 度的线性度

(2) 当前耦合计算状态下组分扩散热流占总热流 的主要部分, 尤其 $\mathrm{CO}_{2}$ 部分影响最大; 所采用的热防护 系统能够有效阻止气动加热向舱内传递, 防隔热性能 良好; 防热大底气动加热与表面辐射散热之间可快速 趋向局部热辐射平衡, 工程上可基于解耦的流场数值 模拟并采用辐射平衡壁面条件近似获得符合真实物理 的气动加热规律.

(3) 气固界面热化学对耦合热效应的影响讨论认 为, 完全非催化/完全催化壁面热流常规性地随耦合时 间逐渐降低, 但有限速率催化热流因界面非平衡热化 学效应而先增后降; 壁面热流与壁温的线性度因界面 非平衡热化学所致扩散热流的引入而减弱.

\section{参考文献}

1 叶培建, 杨孟飞, 彭兢, 等. 中国深空探测进入再入返回技术的发展现状和展望. 中国科学: 技术科学, 2015, 45: 229-238

2 Ye P J, Sun Z Z, Rao W, et al. Mission overview and key technologies of the first Mars probe of China. Sci China Tech Sci, 2017, 60: 649-657

3 Congdon W M, Edquist C T, Henline W D. Thermal protection studies for the 1996 Pathfinder mission to Mars. In: Proceeding of the 32nd Aerospace Sciences Meeting \& Exhibit. Reno, NV, 1994

4 Reynier P. Survey of aerodynamics and aerothermodynamics efforts carried out in the frame of Mars exploration projects. Prog Aerosp Sci, 2014, 70: $1-27$

5 Culler A J, McNamara J J. Studies on fluid-thermal-structural coupling for aerothermoelasticity in hypersonic flow. AIAA J, 2010, 48: 17211738

6 Thornton E A, Dechaumphai P. Coupled flow, thermal, and structural analysis of aerodynamically heated panels. J Aircraft, 1988, 25: 1052-1059 
7 Dechaumphai P, Wieting A R, Thornton E A. Flow-thermal-structural study of aerodynamically heated leading edges. J Spacecraft Rockets, 1989, 26: 201-209

8 Chen Y K, Henline W D, Tauber M E. Mars Pathfinder trajectory based heating and ablation calculations. J Spacecraft Rockets, 1995, 32: 225230

9 Milos F S, Chen Y K, Congdon W M, et al. Mars pathfinder entry temperature data, aerothermal heating, and heatshield material response. J Spacecraft Rockets, 1989, 36: 380-391

10 黄唐, 毛国良, 姜贵庆. 二维流场-热-结构一体化数值模拟. 空气动力学学报, 2000, 18: 115-119

11 耿湘人, 张涵信, 沈清. 高速飞行器流场和固体结构温度场一体化计算新方法的初步研究. 空气动力学学报, 2002, 20: 422-427

12 桂业伟, 袁湘江. 类前缘防热层流场和热响应耦合计算研究. 工程热物理学报, 2002, 23: 733-735

13 Milos F S, Rasky D J. Review of numerical procedures for computational surface thermochemistry. J Thermophys Heat Transf, 1994, 8: 24-34

14 Hirschel E H, Weiland C. Selected Aerothermodynamic Design Problems of Hypersonic Flight Vehicles. Berlin: Springer Press, 2009

15 刘深深, 唐伟, 桂业伟, 等. 一种多场耦合数据传递新方法. 宇航学报, 2016, 37: 61-66

16 杨肖峰, 唐伟, 桂业伟, 等. 火星环境高超声速催化加热特性研究. 宇航学报, 2017, 38: 205-211

17 Wright M J, Tang C Y, Edquist K T, et al. A review of aerothermal modeling for Mars entry missions. In: Proceeding of the 48th AIAA Aerospace Sciences Meeting Including the New Horizons Forum and Aerospace Exposition. Orlando, 2010

18 Suzuki K, Abe T. Thermochemical nonequilibrium viscous shock-layer analysis for a Mars aerocapture vehicle. J Thermophys Heat Transfer, 1994, 8: 773-780

19 Park C, Howe J T, Jaffe R L, et al. Review of chemical-kinetic problems of future NASA missions. II: Mars entries. J Thermophys Heat Transfer, 1994, 8: 9-23

20 Anderson J D Jr. Hypersonic and High-Temperature Gas Dynamics. Reston: American Institute of Aeronautics and Astronautics, 2009

21 Blottner F G, Johnson M, Ellis M. Chemically reacting viscous flow program for multicomponent gas mixtures. Technical Report. Albuquerque: Sandia Laboratories, 1971

22 Wilke C R. A viscosity equation for gas mixtures. J Chem Phys, 1950, 18: 517-519

23 刘否. 高超声速飞行器热气动弹性特性及相似准则研究. 博士学位论文. 绵阳: 中国空气动力研究与发展中心, 2014

24 MacLean M, Wadhams T, Holden M. Investigation of blunt bodies with $\mathrm{CO}_{2}$ test gas including catalytic effects. In: Proceeding of the 38th AIAA Thermophysics Conference. Toronto, 2005

25 Prabhu D K, Saunders D A. On heatshield shapes for Mars entry capsule. In: AIAA Aerospace Sciences Meeting Including the New Horizons Forum \& Aerospace Exposition. Reno, NV, 2002

26 Mitcheltree R A, Gnoffo P A. Wake flow about the Mars Pathfinder entry vehicle. J Spacecraft Rockets, 1999, 32: 771-776

27 Tompkins S S, Bremer W D, Clark R K. An assessment of the readiness of ablative materials for preflight applications to the shuttle orbiter. NASA Technical Report, N80-28418, 1980

28 Marschall J, MacLean M. Finite-rate surface chemistry model, I: Formulation and reaction system examples. In: Proceeding of the 42nd AIAA Thermophysics Conference. Honolulu, 2011 


\title{
Influence of surface catalysis on coupled aerodynamic heating for Mars entries
}

\author{
YANG XiaoFeng ${ }^{1}$, GUI YeWei ${ }^{1,2}$, LIU Lei ${ }^{2}$, TANG $\mathrm{Wei}^{2} \&$ DU YanXia ${ }^{1,2}$ \\ ${ }^{1}$ State Key Laboratory of Aerodynamics, China Aerodynamics Research and Development Center, Mianyang 621000, China; \\ ${ }^{2}$ Conputational Aerodynamics Institute, China Aerodynamics Research and Development Center, Mianyang 621000, China
}

The integral simulation of aerodynamic heating for Mars entries encounters great challenges due to exceptional and complex hightemperature real-gas effect and gas-solid thermochemistry effect. Coupling computation strategy for hypersonic nonequilibrium aerodynamic heating and in-depth heat transfer was established in order to accurately predict the thermal environment of Mars entry vehicles. Coupling analysis for the heatshield of Mars entry vehicle was then performed to reveal the mechanism how the nonequilibrium thermochemistry affects the coupled aerodynamic heating. Computation results show that both the convection and diffusion heatflux from non-equilibrium flow field have large influences on the total aerodynamic heating rate; the diffusion component contributes the foremost to the overall aerodynamic heating, especially for that originating from $\mathrm{CO}_{2}$; the current thermal protection structure can effectively prevent the severe thermal load from transferring into the cabin, and the aerodynamic heating and the surface radiation can rapidly get close to thermal equilibrium, accounting for the validation of the radiation equilibrium assumption for uncoupled aerodynamic heating prediction; the heatflux decreases for non- and fully catalytic walls while the finiterate catalytic heating rises and then drops due to the gas-solid interface thermochemistry effect; the linearity between the surface heatflux and temperature will get weak due to the introduction of diffusion heating induced by the gas-solid interface thermochemistry.

aerodynamic heating, structure heat transfer, coupling computation, Mars entry capsule, surface thermochemistry, catalytic heating

doi: 10.1360/N092017-00228 